\title{
Inference on Downton's Bivariate Exponential Distribution Based on Moving Extreme Ranked Set Sampling
}

\author{
Ahmed Ali Hanandeh ${ }^{1}$ and Mohammad Fraiwan Al-Saleh ${ }^{2}$ \\ ${ }^{1}$ Dept. Mathematical Sciences, University of Cincinnati, Ohio, USA \\ ${ }^{2}$ Dept. Statistics, Yarmouk University, Irbid, Jordan
}

\begin{abstract}
The purpose of this paper is to estimate the parameters of Downton's bivariate exponential distribution using moving extreme ranked set sampling (MERSS). The estimators obtained are compared via their biases and mean square errors to their counterparts using simple random sampling (SRS). Monte Carlo simulations are used whenever analytical comparisons are difficult. It is shown that these estimators based on MERSS with a concomitant variable are more efficient than the corresponding ones using SRS. Also, MERSS with a concomitant variable is easier to use in practice than RSS with a concomitant variable. Furthermore, the best unbiased estimators among all unbiased linear combinations of the MERSS elements are derived for some parameters.
\end{abstract}

Zusammenfassung: Das Ziel dieser Arbeit ist es, die Parameter der Downton bivariaten Exponentialverteilung mittels "moving extreme ranked set sampling” (MERSS) zu schätzen. Die erhaltenen Schätzer werden bezüglich Bias und mittleren quadratischen Fehler mit deren Gegenstücken basierend auf einfache Zufallsstichproben (SRS) verglichen. Monte Carlo Simulationen werden verwendet, wenn analytische Vergleiche schwierig sind. Es wird gezeigt, dass die Schätzer basierend auf MERSS mit einer begleitenden Variablen effizienter sind als die entsprechenden beruhend auf SRS. Auch ist MERSS mit einer begleitenden Variablen einfacher in der Praxis zu verwenden als RSS mit einer begleitenden Variablen. Außerdem werden die besten Schätzer unter allen unverzerrten Linearkombinationen der MERSS Elemente für einige Parameter hergeleitet.

Keywords: Simple Random Sampling, Moving Extreme Ranked Set Sampling, Concomitant Variable.

\section{Introduction}

The most popular sampling method that is usually used in statistical studies is Simple Random Sampling (SRS). A SRS of size $n$ from a population is a subset of the population consisting of $n$ units selected in such a way that all subsets of size $n$ are equally likely to be selected; but for an infinite population (which we are interested in) each unit of the sample is selected independently and comes from the same population (i.i.d.). Simple 
random sampling is the basic building block and point of reference for all other sampling methods.

McIntyre (1952) suggested a sampling technique which was later called Ranked Set Sampling (RSS). This technique of data collection was introduced for situations where taking the actual measurements on sample observations is difficult (i.e., costly, timeconsuming) as compared to the judgment ranking of them. The ranked set sampling technique can be executed as follows:

- Step 1: Randomly draw $m$ simple random samples each of size $m$ from the population of interest.

- Step 2: Within each of the $m$ sets, the sampled items are ranked from lowest to largest according to the variable of interest based on the researcher's judgment or by any negligible cost method that does not require actual quantifications.

- Step 3: From the first set of $m$ units, the unit ranked lowest is measured. From the second set of $m$ units, the unit ranked second lowest is measured. The process is continued until the $m$ th ranked unit is measured from the $m$ th set. Note that $m^{2}$ units are sampled but only $m$ of them are measured with respect to the variable of interest.

The above 3 steps describe one cycle of the RSS technique.

- Step 4: Repeat steps 1 to 3, if necessary, $k$ independent times (cycles) to obtain a total sample of size $n=m k$ units.

In McIntyre's RSS procedure, it is assumed that the researcher could order a set of size $m$ with respect to the characteristic of interest. Many authors recommended that $m$ should be 2, 3, or 4 to minimize the ranking error (see Takahasi and Wakimoto, 1968).

There are several variations of RSS. One of them is Moving Extreme Ranked Set Sampling (MERSS). In this procedure only the maximum (or minimum) of sets of varied size is identified (by judgment) for quantification. The MERSS, as described by Al-Odat and Al-Saleh (2001) and Al-Saleh and Al-Hadrami (2003a, 2003b) can be executed as follows:

- Step 1: Select $m$ simple random samples of sizes $1, \ldots, m$, respectively.

- Step 2: For each of these samples, measure accurately the maximum ordered observation from the first set identified by judgment, the maximum ordered observation from the second set, etc.. The process continues in this way until the maximum ordered observation from the last $m$ th sample is measured.

- Step 3: Repeated steps 1 and 2, if necessary, $r$ times to obtain a sample of size $n=r m$.

\section{Main Results about RSS and its Variations}

RSS was introduced by McIntyre (1952) in the context of estimating pasture yields. He claimed, without providing a mathematical proof, that

1. $\hat{\mu}_{R S S}=\frac{1}{m k} \sum_{r=1}^{k} \sum_{i=1}^{m} X_{[i] r}$ which is the mean of a sample of size $m k$ obtained using the RSS technique, is an unbiased estimator of the population mean $\mu . X_{[i] r}$ is the $i$ th judgment order statistic in the $r$ th cycle.

2. With perfect ranking, the efficiency of RSS w.r.t. SRS in estimation the population mean is nearly $(m+1) / 2$ for typical unimodal distributions. 
3. The efficiency of the estimators of higher population moments based on RSS are only slightly better than those based on SRS.

Takahasi and Wakimoto (1968) obtained the following main theoretical results.

Under perfect ranking, the mean of a RSS is an unbiased estimator of the population mean and its variance is always smaller than the variance of the mean of a SRS of equal size. Also, they showed that

$$
f(x)=\frac{1}{m} \sum_{i=1}^{m} f_{i}(x), \quad \mu=\frac{1}{m} \sum_{i=1}^{m} \mu_{i}
$$

and

$$
\sigma^{2}=\frac{1}{m} \sum_{i=1}^{m} \sigma_{i}^{2}+\frac{1}{m} \sum_{i=1}^{m}\left(\mu_{i}-\mu\right)^{2}
$$

where $f(x)$ denotes the probability density function (pdf) of a random variable $X$ with $\mathrm{E}(X)=\mu$ and $\operatorname{var}(X)=\sigma^{2}$. Furthermore, $f_{i}(x)$ is the pdf of the $i$ th order statistic $X_{(i)}$ with $\mathrm{E}\left(X_{(i)}\right)=\mu_{i}$ and $\operatorname{var}\left(X_{(i)}\right)=\sigma_{i}^{2}$.

The performance of the estimators is assessed using either the relative precision (RP) or the relative saving (RS), which are defined as

$$
\begin{aligned}
& \mathrm{RP}=\operatorname{eff}\left(\hat{\mu}_{R S S}, \hat{\mu}_{S R S}\right)=\frac{\operatorname{var}\left(\hat{\mu}_{S R S}\right)}{\operatorname{var}\left(\hat{\mu}_{R S S}\right)} \\
& \mathrm{RS}=\frac{\operatorname{var}\left(\hat{\mu}_{S R S}\right)-\operatorname{var}\left(\hat{\mu}_{R S S}\right)}{\operatorname{var}\left(\hat{\mu}_{S R S}\right)}=1-\frac{1}{\mathrm{RP}} .
\end{aligned}
$$

They also showed that

$$
\operatorname{var}\left(\hat{\mu}_{R S S}\right)=\frac{1}{m}\left(\sigma^{2}-\frac{1}{m} \sum_{i=1}^{m}\left(\mu_{i}-\mu\right)^{2}\right) \quad \text { and } \quad \mathrm{RS}=\frac{1}{m} \sum_{i=1}^{m}\left(\frac{\mu_{i}-\mu}{\sigma}\right)^{2},
$$

and thus

$$
0 \leq \mathrm{RS} \leq \frac{m-1}{m+1} \quad 1 \leq \operatorname{eff}\left(\hat{\mu}_{R S S}, \hat{\mu}_{S R S}\right) \leq \frac{m+1}{2}
$$

where

$$
\hat{\mu}_{S R S}=\frac{1}{m} \sum_{i=1}^{m} X_{i}
$$

is the mean of a sample of size $m$ obtained using the SRS technique, and

$$
\hat{\mu}_{R S S}=\frac{1}{m k} \sum_{r=1}^{k} \sum_{i=1}^{m} X_{[i] r}
$$

is the mean of a sample of size $m k$ obtained using the RSS technique.

Stokes (1977) studied RSS with concomitant variables. In this case, the variable of interest is difficult to rank but it is related to some other variable that is easy to rank (concomitant variable). We use $(X, Y)$ in the sequel, where $X$ is the variable of interest and 
$Y$ is the concomitant one. Samawi, Ahmed, and Abu-Dayyeh (1996) introduced the Extreme Ranked Set Sampling (ERSS) procedure. It was shown that the ERSS estimator of the mean is more efficient than the usual SRS mean and unbiased if the underlying distribution is symmetric. In this ERSS, only the two extremes (minimum, maximum) are identified by judgment for different sets. Al-Saleh and Al-Kadri (2000) considered double RSS (DRSS) as a procedure that increases the efficiency of RSS estimator without increasing the set size $m$. Al-Saleh and Al-Omari (2002) generalized DRSS to multistage ranked set sampling. They showed that the efficiency is always between 1 and $m^{2}$ for all distributions and equals $m^{2}$ for the uniform distribution when the number of stages goes to infinity. Al-Saleh and Zheng (2002) proposed a new RSS for two characteristics and called it a bivariate ranked set sampling.

MERSS is a useful modification of RSS. Unlike RSS, MERSS allows for an increase of the set size without introducing too much ranking error. MERSS was introduced by Al-Odat and Al-Saleh (2001) who also introduced the concept of varied set size RSS. Al-Saleh and Al-Hadrami (2003a, 2003b) used varied set size of RSS (coined by them MERSS) for estimating the mean of the normal and exponential distribution, and they showed that this procedure could be more useful than SRS for estimating the mean of any symmetric distribution. Al-Saleh and Samawi (2010) estimated the odds based on MERSS. The suggested estimator based on MERSS is motivated by some of the theoretical properties of the sum of geometric series.

RSS and some of its variation were used by many authors in parametric estimation like bivariate normal, exponential, Downton's bivariate exponential (see Downton, 1970), etc..

Moran (1967) introduced a bivariate exponential distribution, which is one of the most important bivariate distributions in reliability. There are various bivariate exponential distributions. In this research, we are interested in Downton's bivariate exponential distribution with pdf

$$
f(x, y)=\frac{1}{\lambda_{1} \lambda_{2}(1-\rho)} \exp \left[-\left(\frac{x}{\lambda_{1}(1-\rho)}+\frac{y}{\lambda_{2}(1-\rho)}\right)\right] I_{0}\left[\frac{2(\rho x y)^{\frac{1}{2}}}{\left(\lambda_{1} \lambda_{2}\right)^{\frac{1}{2}}(1-\rho)}\right],
$$

where $x, y, \lambda_{1}, \lambda_{2}>0,0 \leq \rho<1$ and

$$
I_{0}(z)=\sum_{k=0}^{\infty} \frac{(z / 2)^{2 k}}{k !^{2}}
$$

is the modified Bessel function of the first kind of order zero. If $(X, Y)$ is a bivariate random variable that has Downton's bivariate exponential distribution, then from (1) the marginal distributions of $X$ and $Y$ are exponential with parameters $\lambda_{1}$ and $\lambda_{2}$, respectively. Thus, in particular, $\mathrm{E}(X)=\lambda_{1}$ and $\mathrm{E}(Y)=\lambda_{2}$. Downton (1970) further showed that

$$
\begin{gathered}
\mathrm{E}(Y \mid X=x)=(1-\rho) \lambda_{2}+\rho \frac{\lambda_{2}}{\lambda_{1}} x \\
\operatorname{var}(Y \mid X=x)=(1-\rho)^{2} \lambda_{2}^{2}+2 \rho(1-\rho) \frac{\lambda_{2}^{2}}{\lambda_{1}} x .
\end{gathered}
$$


The parameter $\rho$ is the correlation coefficient between $X$ and $Y$ with independence corresponding to $\rho=0$ since $I_{0}(0)=1$. This distribution is a candidate distribution for positively correlated bivariate exponential data, in which the conditional mean and variance of one variable is increasing function of the other variable.

This distribution has real applications in several fields. Lefebvre (2004) considered forecasting the flow values of the Mistassini river in Quebec (Canada). The relation between oil pollution of sea water and tar deposit near sea shore is another application considered by Bain (1978). In this application, the oil pollution is hard and expensive to measure while the tar deposit can be ranked visually. For more applications, see also Balakrishna and Lai (2009, pp. 401-466).

Iliopoulos (2003) showed that the joint sufficient statistic for the three parameters based on the $\operatorname{SRS}\left(X_{1}, Y_{1}\right), \ldots,\left(X_{m}, Y_{m}\right)$ from this distribution is

$$
\left(X_{1} Y_{1}, \ldots, X_{m} Y_{m}, \sum_{i=1}^{m} X_{i}, \sum_{i=1}^{m} Y_{i}\right) .
$$

Nagao and Kadoya (1971) showed that the maximum likelihood estimator of $\lambda_{1}$ and $\lambda_{2}$ is $\bar{X}$ and $\bar{Y}$, respectively.

Two classes of estimators of the parameter $\rho$ based on the complete bivariate sample were derived in Al-Saadi and Young (1980).

The method of moments estimator is based on the statistic

$$
\hat{\rho}=\frac{\sum_{i=1}^{m} X_{i} Y_{i}}{m \bar{X} \bar{Y}}-1
$$

where $\bar{X}=\frac{1}{m} \sum_{i=1}^{m} X_{i}$ and $\bar{Y}=\frac{1}{m} \sum_{i=1}^{m} Y_{i}$. Using the condition $0 \leq \rho<1$, a modified estimator for $\rho$ is

$$
\hat{\rho}_{1}= \begin{cases}0 & \text { if } \hat{\rho}<0 \\ \hat{\rho} & \text { if } 0 \leq \hat{\rho} \leq 1 \\ 1 & \text { if } \hat{\rho}>1\end{cases}
$$

Another estimator is based on the sample correlation coefficient

$$
r=\frac{\sum_{i=1}^{m}\left(X_{i}-\bar{X}\right)\left(Y_{i}-\bar{Y}\right)}{\sqrt{\sum_{i=1}^{m}\left(X_{i}-\bar{X}\right)^{2} \sum_{i=1}^{m}\left(Y_{i}-\bar{Y}\right)^{2}}} .
$$

Because $0 \leq \rho<1$, they finally suggested to use

$$
\hat{\rho}_{2}= \begin{cases}0 & \text { if }-1 \leq r<0 \\ r & \text { if } r \geq 0\end{cases}
$$

Note that the first estimator is a function of the sufficient statistic, whereas the second estimator is not, so we will use the first estimator in what follows.

Al-Saleh and Diab (2009) estimated the parameters of Downton's bivariate exponential distribution based on a RSS. He and Nagaraja (2011) estimated the correlation coefficient of Downton's bivariate exponential distribution when all other parameters are 
unknown using incomplete samples. For more details about RSS and its variations see Chen, Bai, and Sinha (2004). For more details about this work see Hanadeh (2011).

In this paper, we consider the estimation of the parameters of a $\operatorname{DBED}\left(\lambda_{1}, \lambda_{2}, \rho\right)$ distribution using MERSS. The suggested estimators are compared with the corresponding ones using SRS. Estimation of $\lambda_{1}$ and $\lambda_{2}$ for the two cases of known and unknown correlation coefficient $\rho$ is considered. Furthermore, estimation of the correlation coefficient $\rho$ for the two cases of known and unknown $\lambda_{1}$ and $\lambda_{2}$ is also discussed.

\section{Mean and Variance of $\boldsymbol{X}_{(k: k)}$ and $\boldsymbol{Y}_{[k: k]}$}

Let $\left(X_{1}, Y_{1}\right), \ldots,\left(X_{m}, Y_{m}\right)$ be a random sample from Downton's bivariate exponential distribution, $\operatorname{DBED}\left(\lambda_{1}, \lambda_{2}, \rho\right)$, with common density given in (1). Let $f_{X}(x)$ and $f_{Y}(y)$ be the marginal densities of $X$ and $Y$, respectively, and let $F_{X}(x)$ and $F_{Y}(y)$ be the corresponding cumulative distribution function (cdf) of $X$ and $Y$, respectively.

Now, suppose that $\left\{\left(X_{(1: 1)}, Y_{[1: 1]}\right), \ldots,\left(X_{(k: k)}, Y_{[k: k]}\right)\right\}, k=1, \ldots, m$, is a MERSS sample from the DBED, $X_{(i: m)}$ is the $i$ th order statistic of $X_{1}, \ldots, X_{m}$, and $Y_{[i: m]}$ is the $Y$ variate paired with $X_{(i: k)}$, then $Y_{[i: k]}$ is called a concomitant order statistic. If the judgment ranking on the $X$ variate is perfect, then $\left(X_{(k: k)}, Y_{[k: k]}\right)$ has pdf

$$
f_{X_{(k: k)}, Y_{[k: k]}}(x, y)=f_{X_{(k: k)}}(x) f_{Y \mid X}(y \mid x),
$$

for $k=1, \ldots, m$, where $f_{X_{(k: k)}}(x)$ is the pdf of the $k$ th order statistic of a SRS of size $k$ from an exponential distribution, $f_{Y_{[k: k]}}(x)$ is the pdf of the corresponding variable $Y$ (concomitant order statistic) and $f_{Y \mid X}(y \mid x)$ is the conditional pdf of $(Y \mid X=x)$ (cp. Yang, 1977).

But,

$$
\begin{aligned}
f_{X_{(k: k)}}(x) & =k\left(F_{X}(x)\right)^{k-1} f_{X}(x) \\
& =k\left(1-\exp \left(-\frac{x}{\lambda_{1}}\right)\right)^{k-1} f_{X}(x) .
\end{aligned}
$$

Also,

$$
\begin{aligned}
f_{X_{(k: k)}, Y_{[k: k]}}(x, y) & =\frac{f_{X, Y}(x, y)}{f_{X}(x)} k\left(1-\exp \left(-\frac{x}{\lambda_{1}}\right)\right)^{k-1} f_{X}(x) \\
& =k\left(1-\exp \left(-\frac{x}{\lambda_{1}}\right)\right)^{k-1} f_{X, Y}(x, y) .
\end{aligned}
$$

It can be verified that if $Z_{1}, \ldots, Z_{k}$ are i.i.d. from an $\operatorname{Exponential}\left(\lambda_{1}\right)$ distribution then $X_{(k: k)}$ has the same distribution as $\sum_{j=1}^{k} \frac{Z_{j}}{k-j+1}$ (see Yang, 1977). Thus, it follows that

$$
\begin{aligned}
\mathrm{E}\left(X_{(k: k)}\right) & =\sum_{j=1}^{k} \frac{\mathrm{E}\left(Z_{j}\right)}{k-j+1}=\lambda_{1} \sum_{j=1}^{k} \frac{1}{k-j+1} \\
\operatorname{var}\left(X_{(k: k)}\right) & =\sum_{j=1}^{k} \frac{\operatorname{var}\left(Z_{j}\right)}{(k-j+1)^{2}}=\lambda_{1}^{2} \sum_{j=1}^{k} \frac{1}{(k-j+1)^{2}} .
\end{aligned}
$$


Moreover, we have

$$
\begin{aligned}
\mathrm{E}\left(Y_{[k: k]}\right) & =\mathrm{E}\left(\mathrm{E}\left(Y_{[k: k]} \mid X_{(k: k)}\right)\right) \\
& =(1-\rho) \lambda_{2}+\rho \frac{\lambda_{2}}{\lambda_{1}} \mathrm{E}\left(X_{(k: k)}\right) .
\end{aligned}
$$

Using (5) we get

$$
\mathrm{E}\left(Y_{[k: k]}\right)=(1-\rho) \lambda_{2}+\rho \lambda_{2} \sum_{j=1}^{k} \frac{1}{k-j+1}
$$

Also,

$$
\operatorname{var}\left(Y_{[k: k]}\right)=\mathrm{E}\left(\operatorname{var}\left(Y_{[k: k]} \mid X_{(k: k)}\right)\right)+\operatorname{var}\left(\mathrm{E}\left(Y_{[k: k]} \mid X_{(k: k)}\right)\right)
$$

Hence,

$$
\operatorname{var}\left(Y_{[k: k]}\right)=\lambda_{2}^{2}\left\{(1-\rho)^{2}+2 \rho(1-\rho) \sum_{j=1}^{k} \frac{1}{k-j+1}+\rho^{2} \sum_{j=1}^{k} \frac{1}{(k-j+1)^{2}}\right\} .
$$

\section{Unbiased Estimation of $\lambda_{1}, \lambda_{2}$ with Known $\rho$}

We now consider the estimation of $\lambda_{1}$ and $\lambda_{2}$ when $\rho$ is known using both MERSS and SRS. Then the performance of the suggested estimators is investigated.

Denote the unbiased estimator of $\lambda_{1}$ and $\lambda_{2}$ based on SRS by $\hat{\lambda}_{1 S R S}$ and $\hat{\lambda}_{2 S R S}$, respectively, and those based on MERSS by $\hat{\lambda}_{1 M E R S S}$ and $\hat{\lambda}_{2 M E R S S}$, respectively, with

$$
\hat{\lambda}_{1 S R S}=\frac{1}{m} \sum_{i=1}^{m} X_{i} \quad \text { and } \quad \hat{\lambda}_{1 M E R S S}=\frac{1}{S_{m}} \sum_{k=1}^{m} X_{(k: k)}
$$

where

$$
S_{m}=\sum_{k=1}^{m} \frac{m+1-k}{k}
$$

Now

$$
\operatorname{var}\left(\hat{\lambda}_{1 M E R S S}\right)=\frac{C_{m}}{S_{m}^{2}} \lambda_{1}^{2}
$$

where

$$
C_{m}=\sum_{k=1}^{m} \frac{(m+1)-k}{k^{2}}
$$

Hence,

$$
\begin{aligned}
\operatorname{eff}\left(\hat{\lambda}_{1 M E R S S}, \hat{\lambda}_{1 S R S}\right) & =\frac{\operatorname{var}\left(\hat{\lambda}_{1 S R S}\right)}{\operatorname{var}\left(\hat{\lambda}_{1 M E R S S}\right)} \\
& =\frac{S_{m}^{2}}{m C_{m}} \\
& =\sum_{k=1}^{m} \frac{(m+1)-k}{m k} \frac{\sum_{k=1}^{m} \frac{(m+1)-k}{k}}{\sum_{k=1}^{m} \frac{(m+1)-k}{k^{2}}},
\end{aligned}
$$


which is clearly larger than 1 and increasing in $m$.

Also,

$$
\hat{\lambda}_{2 S R S}=\frac{1}{m} \sum_{i=1}^{m} Y_{i} \quad \text { and } \quad \hat{\lambda}_{2 M E R S S}=\frac{1}{m(1-\rho)+\rho S_{m}} \sum_{k=1}^{m} Y_{[k: k]} .
$$

It follows that

$$
\begin{array}{r}
\operatorname{var}\left(\hat{\lambda}_{2 M E R S S}\right)=\frac{1}{\left(m(1-\rho)+\rho S_{m}\right)^{2}} \sum_{k=1}^{m}\left\{(1-\rho) \lambda_{2}^{2}\left((1-\rho)+2 \rho \sum_{j=1}^{k} \frac{1}{k-j+1}\right)\right. \\
\left.+\rho^{2} \lambda_{2}^{2} \sum_{j=1}^{k} \frac{1}{(k-j+1)^{2}}\right\},
\end{array}
$$

which can be simplified to

$$
\operatorname{var}\left(\hat{\lambda}_{2 M E R S S}\right)=\frac{\lambda_{2}^{2}}{\left(m(1-\rho)+\rho S_{m}\right)^{2}}\left(m(1-\rho)^{2}+2 \rho(1-\rho) S_{m}+\rho^{2} C_{m}\right)
$$

Hence, using (12) and (13) we get

$$
\begin{aligned}
\operatorname{eff}\left(\hat{\lambda}_{2 M E R S S}, \hat{\lambda}_{2 S R S}\right) & =\frac{\operatorname{var}\left(\hat{\lambda}_{2 S R S}\right)}{\operatorname{var}\left(\hat{\lambda}_{2 M E R S S}\right)} \\
& =\frac{\left(m(1-\rho)+\rho S_{m}\right)^{2}}{m\left(m(1-\rho)^{2}+2 \rho(1-\rho) S_{m}+\rho^{2} C_{m}\right)},
\end{aligned}
$$

which is also clearly larger than 1 , since

$\operatorname{eff}\left(\hat{\lambda}_{2 M E R S S}, \hat{\lambda}_{2 S R S}\right)=\frac{m^{2}(1-\rho)^{2}+2 m \rho(1-\rho) S_{m}+\rho^{2} S_{m}^{2}}{m^{2}(1-\rho)^{2}+2 m \rho(1-\rho) S_{m}+m \rho^{2} C_{m}} \geq 1 \Leftrightarrow \frac{S_{m}^{2}}{m C_{m}} \geq 1$

Table 1 contains efficiencies of $\hat{\lambda}_{1 M E R S S}$ and $\hat{\lambda}_{2 M E R S S}$ with respect to the respective estimators using SRS. Based on Table 1 we conclude that

- $\operatorname{eff}\left(\hat{\lambda}_{1 M E R S S}, \hat{\lambda}_{1 S R S}\right)$ and $\operatorname{eff}\left(\hat{\lambda}_{2 M E R S S}, \hat{\lambda}_{2 S R S}\right)$ are always greater than 1.

- $\operatorname{eff}\left(\hat{\lambda}_{1 M E R S S}, \hat{\lambda}_{1 S R S}\right)$ is fixed in $\rho$ and increasing in $m$.

- $\operatorname{eff}\left(\hat{\lambda}_{2 M E R S S}, \hat{\lambda}_{2 S R S}\right)$ increases in $m$ for fixed $\rho$ and increases in $\rho$ for fixed $m$.

- $\operatorname{eff}\left(\hat{\lambda}_{2 M E R S S}, \hat{\lambda}_{2 S R S}\right) \rightarrow \operatorname{eff}\left(\hat{\lambda}_{1 M E R S S}, \hat{\lambda}_{1 S R S}\right)$ for any $m$ as $\rho \rightarrow 1$. 
Table 1: $\operatorname{eff}\left(\hat{\lambda}_{1 M E R S S}, \hat{\lambda}_{1 S R S}\right)\left(\operatorname{eff}\left(\hat{\lambda}_{2 M E R S S}, \hat{\lambda}_{2 S R S}\right)\right)$

\begin{tabular}{ccccc}
\hline \multicolumn{5}{c}{$m$} \\
$\rho$ & 2 & 3 & 4 & 5 \\
\hline 0.1 & $1.389(1.004)$ & $1.733(1.008)$ & $2.044(1.012)$ & $2.330(1.015)$ \\
0.2 & $1.389(1.016)$ & $1.733(1.031)$ & $2.044(1.044)$ & $2.330(1.055)$ \\
0.3 & $1.389(1.035)$ & $1.733(1.066)$ & $2.044(1.093)$ & $2.330(1.116)$ \\
0.4 & $1.389(1.061)$ & $1.733(1.113)$ & $2.044(1.158)$ & $2.330(1.197)$ \\
0.5 & $1.389(1.095)$ & $1.733(1.173)$ & $2.044(1.241)$ & $2.330(1.299)$ \\
0.6 & $1.389(1.135)$ & $1.733(1.247)$ & $2.044(1.342)$ & $2.330(1.425)$ \\
0.7 & $1.389(1.184)$ & $1.733(1.336)$ & $2.044(1.467)$ & $2.330(1.581)$ \\
0.8 & $1.389(1.241)$ & $1.733(1.444)$ & $2.044(1.619)$ & $2.330(1.774)$ \\
0.9 & $1.389(1.309)$ & $1.733(1.574)$ & $2.044(1.808)$ & $2.330(2.017)$ \\
\hline
\end{tabular}

\section{Optimal Estimator of $\lambda_{1}, \lambda_{2}$ Based on MERSS Ele- ments}

If $\tau=\left\{\hat{\theta}_{1}, \ldots, \hat{\theta}_{N}\right\}$ is a set of $N$ unbiased estimators of the parameter $\theta$ with $\operatorname{var}\left(\hat{\theta}_{i}\right)=\delta_{i}^{2}$ and $\operatorname{cov}\left(\hat{\theta}_{i}, \hat{\theta}_{j}\right)=0$ for all $i \neq j$, then among all unbiased linear combinations $\sum_{i=1}^{N} b_{i} \hat{\theta}_{i}$, where $b_{i}$ is a constant,

has the smallest variance

$$
\hat{\theta}^{*}=\frac{\sum_{i=1}^{N} \hat{\theta}_{i} / \delta_{i}^{2}}{\sum_{i=1}^{N} 1 / \delta_{i}^{2}}
$$

$$
\operatorname{var}\left(\hat{\theta}^{*}\right)=\frac{1}{\sum_{i=1}^{N} 1 / \delta_{i}^{2}}
$$

(see e.g. Casella and Berger, 2002, p. 363). Using this result, we can find the best unbiased estimators for $\lambda_{1}$ and $\lambda_{2}$ among all unbiased linear combinations of the MERSS elements.

For simplicity, let

$$
a_{k}=\sum_{j=1}^{k} \frac{1}{k-j+1} \quad \text { and } \quad b_{k}=\sum_{j=1}^{k} \frac{1}{(k-j+1)^{2}} .
$$

We know from (5) and (6) that

$$
\mathrm{E}\left(X_{(k: k)}\right)=a_{k} \lambda_{1} \quad \text { and } \quad \operatorname{var}\left(X_{(k: k)}\right)=b_{k} \lambda_{1}^{2} .
$$

Thus, the best unbiased estimator for $\lambda_{1}$ among all unbiased linear combinations of $X_{(k: k)}$ is

$$
T^{*}=\frac{\sum_{k=1}^{m} \frac{X_{(k: k)}}{a_{k}} \frac{a_{k}^{2}}{\lambda_{1}^{2} b_{k}}}{\sum_{k=1}^{m} \frac{a_{k}^{2}}{\lambda_{1}^{2} b_{k}}}=\frac{\sum_{k=1}^{m} \frac{a_{k} X_{(k: k)}}{b_{k}}}{\sum_{k=1}^{m} \frac{a_{k}^{2}}{b_{k}}} .
$$


It follows that

$$
\operatorname{var}\left(T^{*}\right)=\frac{\lambda_{1}^{2}}{\sum_{k=1}^{m} \frac{a_{k}^{2}}{b_{k}}}
$$

Hence, using (16) we get

$$
\operatorname{eff}\left(T^{*}, \hat{\lambda}_{1 S R S}\right)=\frac{\operatorname{var}\left(\hat{\lambda}_{1 S R S}\right)}{\operatorname{var}\left(T^{*}\right)}=\frac{\lambda_{1}^{2}}{m} \frac{1}{\lambda_{1}^{2}} \sum_{k=1}^{m} \frac{a_{k}^{2}}{b_{k}}=\frac{1}{m} \sum_{k=1}^{m}\left[\frac{\left(\sum_{j=1}^{k} \frac{1}{(k-j+1)}\right)^{2}}{\left(\sum_{j=1}^{k} \frac{1}{(k-j+1)^{2}}\right)}\right]
$$

Table 2 gives values of the efficiencies eff $\left(T^{*}, \hat{\lambda}_{1 S R S}\right)$ and eff $\left(\hat{\lambda}_{1 M E R S S}, \hat{\lambda}_{1 S R S}\right)$. Based on these results, we conclude that $\operatorname{eff}\left(T^{*}, \hat{\lambda}_{1 S R S}\right)$ is always larger than 1 . It is larger than $\operatorname{eff}\left(\hat{\lambda}_{1 M E R S S}, \hat{\lambda}_{1 S R S}\right)$ for all values of $m$, i.e. $T^{*}$ is more efficient than $\hat{\lambda}_{1 M E R S S}$ and hence than $\hat{\lambda}_{1 R S S}$ and $\hat{\lambda}_{1 S R S}$. Note that the best linear unbiased estimator and the traditional one, though different, are relatively close to each other.

Table 2: Efficiencies eff $\left(T^{*}, \hat{\lambda}_{1 S R S}\right)$ and $\operatorname{eff}\left(\hat{\lambda}_{1 M E R S S}, \hat{\lambda}_{1 S R S}\right)$

\begin{tabular}{ccc}
\hline$m$ & $\operatorname{eff}\left(T^{*}, \hat{\lambda}_{1 S R S}\right)$ & $\operatorname{eff}\left(\hat{\lambda}_{1 M E R S S}, \hat{\lambda}_{1 S R S}\right)$ \\
\hline 2 & 1.400 & 1.389 \\
3 & 1.756 & 1.733 \\
4 & 2.080 & 2.044 \\
5 & 2.376 & 2.330 \\
\hline
\end{tabular}

Similarly, the best unbiased estimator of $\lambda_{2}$ among all unbiased linear combinations of $Y_{[k: k]}$ is

$$
\omega^{*}=\frac{\sum_{k=1}^{m} \frac{\left(1-\rho+\rho a_{k}\right) Y_{[k: k]}}{\lambda_{1}^{2}\left[(1-\rho)^{2}+2 \rho(1-\rho) a_{k}+\rho^{2} b_{k}\right]}}{\sum_{k=1}^{m} \frac{\left(1-\rho+\rho a_{k}\right)^{2}}{\lambda_{1}^{2}\left[(1-\rho)^{2}+2 \rho(1-\rho) a_{k}+\rho^{2} b_{k}\right]}}
$$

It follows that

$$
\operatorname{var}\left(\omega^{*}\right)=\frac{\lambda_{2}^{2} \sum_{k=1}^{m} \frac{\left(1-\rho+\rho a_{k}\right)^{2}}{(1-\rho)^{2}+2 \rho(1-\rho) a_{k}+\rho^{2} b_{k}}}{\left(\sum_{k=1}^{m} \frac{\left(1-\rho+\rho a_{k}\right)^{2}}{(1-\rho)^{2}+2 \rho(1-\rho) a_{k}+\rho^{2} b_{k}}\right)^{2}} .
$$


Hence, using (19) we get

$$
\begin{aligned}
\operatorname{eff}\left(\omega^{*}, \hat{\lambda}_{2 S R S}\right) & =\frac{\operatorname{var}\left(\hat{\lambda}_{2 S R S}\right)}{\operatorname{var}\left(\omega^{*}\right)} \\
& =\frac{\left(\sum_{k=1}^{m} \frac{\left(1-\rho+\rho a_{k}\right)^{2}}{(1-\rho)^{2}+2 \rho(1-\rho) a_{k}+\rho^{2} b_{k}}\right)^{2}}{m \sum_{k=1}^{m} \frac{\left(1-\rho+\rho a_{k}\right)^{2}}{(1-\rho)^{2}+2 \rho(1-\rho) a_{k}+\rho^{2} b_{k}}}
\end{aligned}
$$

Table 3 includes the efficiencies of $\omega^{*}$ compared to $\hat{\lambda}_{2 S R S}\left(\hat{\lambda}_{2 M E R S S}\right.$ compared to $\left.\hat{\lambda}_{2 S R S}\right)$. Based on Table 3 we conclude that $\operatorname{eff}\left(\omega^{*}, \hat{\lambda}_{2 S R S}\right)$ is always larger than 1. It is increasing in $m$ for fixed $\rho$ and also increasing in $\rho$ for fixed $m$. eff $\left(\omega^{*}, \hat{\lambda}_{2 S R S}\right)$ is larger than $\operatorname{eff}\left(\hat{\lambda}_{2 M E R S S}, \hat{\lambda}_{2 S R S}\right)$ for any size $m$ and $\rho$, i.e., $\omega^{*}$ is more efficient than $\hat{\lambda}_{2 M E R S S}$ and hence than $\hat{\lambda}_{2 R S S}$ and $\hat{\lambda}_{2 S R S}$.

Table 3: $\operatorname{eff}\left(\omega^{*}, \hat{\lambda}_{2 S R S}\right)\left(\operatorname{eff}\left(\hat{\lambda}_{2 M E R S S}, \hat{\lambda}_{2 S R S}\right)\right)$

\begin{tabular}{ccccc}
\hline & \multicolumn{3}{c}{$m$} \\
$\rho$ & 2 & 3 & 4 & 5 \\
\hline 0.1 & $1.005(1.004)$ & $1.009(1.008)$ & $1.013(1.012)$ & $1.016(1.015)$ \\
0.2 & $1.017(1.016)$ & $1.032(1.031)$ & $1.046(1.044)$ & $1.057(1.055)$ \\
0.3 & $1.037(1.035)$ & $1.068(1.066)$ & $1.095(1.093)$ & $1.119(1.116)$ \\
0.4 & $1.063(1.061)$ & $1.115(1.113)$ & $1.160(1.158)$ & $1.199(1.197)$ \\
0.5 & $1.095(1.095)$ & $1.174(1.173)$ & $1.241(1.241)$ & $1.300(1.299)$ \\
0.6 & $1.135(1.135)$ & $1.247(1.247)$ & $1.342(1.342)$ & $1.425(1.425)$ \\
0.7 & $1.184(1.184)$ & $1.337(1.336)$ & $1.467(1.467)$ & $1.582(1.581)$ \\
0.8 & $1.242(1.241)$ & $1.446(1.444)$ & $1.623(1.619)$ & $1.780(1.774)$ \\
0.9 & $1.313(1.309)$ & $1.583(1.574)$ & $1.821(1.808)$ & $2.035(2.017)$ \\
\hline
\end{tabular}

\section{Estimation of $\rho$ with Known $\lambda_{1}, \lambda_{2}$}

Now we consider the estimation of $\rho$ when $\lambda_{1}$ and $\lambda_{2}$ are known using MERSS and SRS. Diab (2006) (see also Al-Saleh and Diab, 2009) derived the following formula for the variance of $\hat{\rho}$ based on SRS:

$$
\operatorname{var}\left(\hat{\rho}_{1 S R S}\right)=\frac{3 \rho^{2}+14 \rho+3}{m}
$$

where

$$
\hat{\rho}_{1 S R S}=\frac{1}{m \lambda_{1} \lambda_{2}} \sum_{i=1}^{m} X_{i} Y_{i}-1
$$


Now, suppose that $\lambda_{1}$ and $\lambda_{2}$ are known, then

$$
\begin{aligned}
\mathrm{E}\left(X_{(k: k)} Y_{[k: k]}\right) & =\mathrm{E}\left[\mathrm{E}\left(X_{(k: k)} Y_{[k: k]} \mid X_{(k: k)}\right)\right]=\mathrm{E}\left[X_{(k: k)} \mathrm{E}\left(Y_{[k: k]} \mid X_{(k: k)}\right)\right] \\
& =\mathrm{E}\left[X_{(k: k)}\left((1-\rho) \lambda_{2}+\rho \frac{\lambda_{2}}{\lambda_{1}} X_{(k: k)}\right)\right] \\
& =(1-\rho) \lambda_{2} \lambda_{1} \sum_{j=1}^{k} \frac{1}{k-j+1}+\rho \frac{\lambda_{2}}{\lambda_{1}} \mathrm{E}\left(X_{(k: k)}^{2}\right) .
\end{aligned}
$$

But,

$$
\begin{aligned}
\mathrm{E}\left(X_{(k: k)}^{2}\right) & =\operatorname{var}\left(X_{(k: k)}\right)+\left[\mathrm{E}\left(X_{(k: k)}\right)\right]^{2} \\
& =\lambda_{1}^{2} \sum_{j=1}^{k} \frac{1}{(k-j+1)^{2}}+\lambda_{1}^{2}\left[\sum_{j=1}^{k} \frac{1}{k-j+1}\right]^{2} .
\end{aligned}
$$

Thus,

$$
\begin{aligned}
\mathrm{E}\left(X_{(k: k)} Y_{[k: k]}\right)= & (1-\rho) \lambda_{2} \lambda_{1} \sum_{j=1}^{k} \frac{1}{k-j+1} \\
& +\rho \frac{\lambda_{2}}{\lambda_{1}}\left(\lambda_{1}^{2} \sum_{j=1}^{k} \frac{1}{(k-j+1)^{2}}+\lambda_{1}^{2}\left[\sum_{j=1}^{k} \frac{1}{k-j+1}\right]^{2}\right) \\
= & \lambda_{1} \lambda_{2}\left(\sum_{j=1}^{k} \frac{1}{k-j+1}\right. \\
& \left.+\rho\left(\sum_{j=1}^{k} \frac{1}{(k-j+1)^{2}}-\sum_{j=1}^{k} \frac{1}{k-j+1}+\left[\sum_{j=1}^{k} \frac{1}{k-j+1}\right]^{2}\right)\right)
\end{aligned}
$$

Hence,

$$
\mathrm{E}\left(\sum_{k=1}^{m} X_{(k: k)} Y_{[k: k]}\right)=\rho \lambda_{1} \lambda_{2}\left(C_{m}-S_{m}+\sum_{k=1}^{m}\left[\sum_{j=1}^{k} \frac{1}{k-j+1}\right]^{2}\right)+\lambda_{1} \lambda_{2} S_{m} .
$$

Thus,

Hence,

$$
\rho=\frac{\mathrm{E}\left(\sum_{k=1}^{m} X_{(k: k)} Y_{[k: k]}\right)-\lambda_{1} \lambda_{2} S_{m}}{\lambda_{1} \lambda_{2}\left(C_{m}-S_{m}+\sum_{k=1}^{m}\left[\sum_{j=1}^{k} \frac{1}{k-j+1}\right]^{2}\right)}
$$

$$
\hat{\rho}_{1 M E R S S}=\frac{\frac{1}{\lambda_{1} \lambda_{2}} \sum_{k=1}^{m} X_{(k: k)} Y_{[k: k]}-S_{m}}{C_{m}-S_{m}+\sum_{k=1}^{m}\left[\sum_{j=1}^{k} \frac{1}{k-j+1}\right]^{2}}=\frac{\frac{1}{\lambda_{1} \lambda_{2}} \sum_{k=1}^{m} X_{(k: k)} Y_{[k: k]}-S_{m}}{C_{m}-S_{m}+\sum_{k=1}^{m} a_{k}^{2}}
$$


is an unbiased estimator of $\rho$. It follows that

$$
\operatorname{var}\left(\hat{\rho}_{1 M E R S S}\right)=\frac{\sum_{k=1}^{m} \operatorname{var}\left(X_{(k: k)} Y_{[k: k]}\right)}{\lambda_{1}^{2} \lambda_{2}^{2}\left(C_{m}-S_{m}+\sum_{k=1}^{m} a_{k}^{2}\right)^{2}}
$$

Thus,

$$
\begin{aligned}
\operatorname{var}\left(\hat{\rho}_{1 M E R S S}\right)= & \left(C_{m}-S_{m}+\sum_{k=1}^{m} a_{k}^{2}\right)^{-2}\left\{2(1-\rho)^{2} \sum_{k=1}^{m}\left(b_{k}+a_{k}^{2}\right)\right. \\
& +24(1-\rho) \rho\left(\sum_{k=1}^{m} k \sum_{j=0}^{k-1}\left(\begin{array}{c}
k-1 \\
j
\end{array}\right) \frac{(-1)^{j}}{(1+j)^{4}}\right) \\
& \left.+\rho^{2}\left(24 \sum_{k=1}^{m} k \sum_{j=0}^{k-1}\left(\begin{array}{c}
k-1 \\
j
\end{array}\right) \frac{(-1)^{j}}{(1+j)^{5}}\right)-\sum_{k=1}^{m}\left[(1-\rho) a_{k}+\rho\left(b_{k}+a_{k}^{2}\right)\right]^{2}\right\} .
\end{aligned}
$$

Hence, we finally get

$$
\operatorname{eff}\left(\hat{\rho}_{1 M E R S S}, \hat{\rho}_{1 S R S}\right)=\frac{\left(3 \rho^{2}+14 \rho+3\right) / m}{\operatorname{var}\left(\hat{\rho}_{1 M E R S S}\right)}
$$

Table 4 contains the efficiencies of $\hat{\rho}_{1 M E R S S}$ w.r.t. $\hat{\rho}_{1 S R S}$. We conclude that the efficiency of $\hat{\rho} 1 M E R S S$ w.r.t. $\hat{\rho}_{1 S R S}$ is always larger than 1 and it is increasing in $m$ for fixed $\rho$.

Table 4: $\operatorname{eff}\left(\hat{\rho}_{1 M E R S S}, \hat{\rho}_{1 S R S}\right)$

\begin{tabular}{ccccc}
\hline & \multicolumn{4}{c}{$m$} \\
$\rho$ & 2 & 3 & 4 & 5 \\
\hline 0.1 & 1.700 & 2.404 & 3.091 & 3.752 \\
0.2 & 1.681 & 2.359 & 3.014 & 3.644 \\
0.3 & 1.670 & 2.335 & 2.977 & 3.591 \\
0.4 & 1.664 & 2.322 & 2.958 & 3.565 \\
0.5 & 1.661 & 2.316 & 2.948 & 3.555 \\
0.6 & 1.659 & 2.312 & 2.945 & 3.553 \\
0.7 & 1.658 & 2.312 & 2.946 & 3.556 \\
0.8 & 1.658 & 2.313 & 2.950 & 3.564 \\
0.9 & 1.658 & 2.315 & 2.955 & 3.573 \\
0.99 & 1.659 & 2.317 & 2.961 & 3.583 \\
\hline
\end{tabular}




\section{Estimation of $\rho$ with Unknown $\lambda_{1}, \lambda_{2}$}

Suppose that $\lambda_{1}$ and $\lambda_{2}$ are unknown, then we can replace them in $\hat{\rho}_{1 S R S}$ by $\hat{\lambda}_{1 S R S}$ and $\hat{\lambda}_{2 S R S}$ to get

$$
\hat{\rho}_{2 S R S}=\frac{1}{m \hat{\lambda}_{1 S R S} \hat{\lambda}_{2 S R S}} \sum_{i=1}^{m} X_{i} Y_{i}-1 .
$$

Since $0 \leq \rho<1$, we modify this estimator by

$$
\hat{\rho}_{2 S R S}^{*}= \begin{cases}0 & \text { if } \hat{\rho}_{2 S R S}<0 \\ \hat{\rho}_{2 S R S} & \text { if } 0 \leq \hat{\rho}_{2 S R S} \leq 1 \\ 1 & \text { if } \hat{\rho}_{2 S R S}>1\end{cases}
$$

Similarly,

$$
\hat{\rho}_{2 M E R S S}=\frac{\frac{\sum_{k=1}^{m} X_{(k: k)} Y_{[k: k]}}{\hat{\lambda}_{1 M E R S S} \hat{\lambda}_{2 M E R S S}^{*}}-S_{m}}{C_{m}-S_{m}+\sum_{k=1}^{m} a_{k}^{2}},
$$

where $\hat{\lambda}_{2 M E R S S}^{*}=\frac{1}{m} \sum_{k=1}^{m} Y_{[k: k]}$ since $\hat{\lambda}_{2 M E R S S}$ depends on $\rho$. Since $0 \leq \rho<1$ we modify this estimator by

$$
\hat{\rho}_{2 M E R S S}^{*}= \begin{cases}0 & \text { if } \hat{\rho}_{2 M E R S S}<0 \\ \hat{\rho}_{2 M E R S S} & \text { if } 0 \leq \hat{\rho}_{2 M E R S S} \leq 1 \\ 1 & \text { if } \hat{\rho}_{2 M E R S S}>1\end{cases}
$$

We compare the bias and the mean squared error (MSE) of the two estimators $\hat{\rho}_{2 M E R S S}^{*}$ and $\hat{\rho}_{2 S R S}^{*}$ by simulation using 10000 repetitions. To simulate a pair $(X, Y)$ from the $\operatorname{DBED}\left(\lambda_{1}, \lambda_{2}, \rho\right)$, Iliopoulos (2003) rewrote the pdf of the DBED (1) as an infinite mixture of independent gamma distributions with geometric mixing weights (see also Diab, 2006; Al-Saleh and Diab, 2009)

$$
f(x, y)=\sum_{k=0}^{\infty} \pi(k ; \rho) \Gamma_{(k+1)}\left(x ; \lambda_{1}(1-\rho)\right) \Gamma_{(k+1)}\left(y ; \lambda_{2}(1-\rho)\right),
$$

where $\Gamma_{\alpha}(\cdot ; \beta)$ is the pdf of the $\operatorname{Gamma}(\alpha, \beta)$ distribution and $\pi(k ; \rho)=(1-\rho) \rho^{k}$, $k=0,1, \ldots$ is the geometric probability function. Let $K$ be a random variable with this geometric pdf. Then, $X$ and $Y$ are conditionally (given $K=k$ ) independent gamma random variables with $\alpha=k+1$ and $\beta_{1}=\lambda_{1}(1-\rho), \beta_{2}=\lambda_{2}(1-\rho)$, respectively. Also, the unconditional distribution of $(X, Y)$ is a $\operatorname{DBED}\left(\lambda_{1}, \lambda_{2}, \rho\right)$. The algorithm of simulating observations form the DBED consists of the following steps:

1. Given $\lambda_{1}, \lambda_{2}$ and $\rho$, simulate $m$ random numbers $K_{i}$ from the geometric distribution with $p=(1-\rho)$. 
2. Given $K_{i}=k_{i}$, simulate two independent random variable $X_{i}$ and $Y_{i}$, where $X_{i}$ is from $\operatorname{Gamma}\left(k_{i}+1 ; \lambda_{1}(1-\rho)\right)$ and $Y_{i}$ is from $\operatorname{Gamma}\left(k_{i}+1 ; \lambda_{2}(1-\rho)\right)$.

3. Use the pairs $\left(X_{i}, Y_{i}\right)$ just generated to compute the values of $\hat{\rho}_{S R S}^{*}$ and $\hat{\rho}_{2 M E R S S}^{*}$.

4. Repeat steps 1 to 3 a 10000 times to get 10000 values of $\hat{\rho}_{2 S R S}^{*}$ and $\hat{\rho}_{2 M E R S S}^{*}$.

Bias and MSE of each of the estimators $\hat{\rho}_{2 S R S}^{*}$ and $\hat{\rho}_{2 M E R S S}^{*}$ are given in Tables 5 and 6 , respectively, and the efficiency of $\hat{\rho}_{2 M E R S S}^{*}$ w.r.t. $\hat{\rho}_{2 S R S}^{*}$ is given in Table 7 .

Based on Tables 5, 6, and 7 we conclude that the efficiency of $\hat{\rho}_{2 M E R S S}^{*}$ w.r.t. $\hat{\rho}_{2 S R S}^{*}$ is larger than 1 for $\rho \leq 0.4$. If $\rho>0.4$ then $\hat{\rho}_{2 M E R S S}^{*}$ is not recommended.

\section{Estimation of $\lambda_{1}, \lambda_{2}$ with Unknown $\rho$}

We noticed that the formula of $\hat{\lambda}_{1 M E R S S}=\frac{1}{S_{m}} \sum_{k=1}^{m} X_{(k: k)}$ does not depend on $\rho$, while $\hat{\lambda}_{2 M E R S S}$ does. Suppose that $\rho$ is unknown, then we can replace it by $\hat{\rho}_{2 M E R S S}^{*}$ in the formula of $\hat{\lambda}_{2 M E R S S}$ to get

$$
\hat{\lambda}_{2 M E R S S}^{*}=\frac{1}{m\left(1-\hat{\rho}_{2 M E R S S}^{*}\right)+\hat{\rho}_{2 M E R S S}^{*} S_{m}} \sum_{k=1}^{m} Y_{[k: k]} .
$$

Bias and MSE of the estimators $\hat{\lambda}_{2 S R S}$ and $\hat{\lambda}_{2 M E R S S}^{*}$ are given in Tables 8 and 9 , respectively, and the efficiency values of $\hat{\lambda}_{2 M E R S S}^{*}$ w.r.t. $\hat{\lambda}_{2 S R S}$ are contained in Table 10. Based on Tables 8,9 , and 10 we conclude that

1. The bias of $\hat{\lambda}_{2 M E R S S}^{*}$ is larger than that of $\hat{\lambda}_{2 S R S}$ as $\rho$ is getting large.

2. The MSE of $\hat{\lambda}_{2 M E R S S}^{*}$ behaves similarly; it is larger than that of $\hat{\lambda}_{2 S R S}$ for most of the cases that we considered.

3. The Estimators based on SRS are more efficient than those based on MERSS for $\rho \geq 0.20$. The suggested estimator is not recommended if some prior information suggests that the unknown $\rho$ is not very small. The reason could be that the estimator of $\rho$ is not a suitable one.

\section{Conclusions}

Moving extreme ranked set sampling is a useful variation of ranked set sampling and more applicable since it allows for an increase of set size without introducing extra ranking errors. In this procedure, only the two extreme values (maximum or minimum) of sets of varied sizes were identified (by judgment) for quantification. In this paper, the main goal was to estimate the parameters of Downton's bivariate exponential distribution using MERSS with concomitant variable. It was assumed that $X$ can be ranked visually while $Y$ was highly correlated with $X$. It was shown that the use of MERSS with concomitant variable gives more efficient estimators for the parameters of Downton's bivariate exponential distribution than the corresponding ones using Simple Random Sample. 
Table 5: Bias of $\hat{\rho}_{2 S R S}^{*}\left(\hat{\rho}_{2 M E R S S}^{*}\right)$

\begin{tabular}{ccccc}
\hline \multicolumn{5}{c}{$m$} \\
$\rho$ & 2 & 3 & 4 & 5 \\
\hline 0.1 & $0.037(0.010)$ & $0.058(0.001)$ & $0.059(-0.012)$ & $0.055(-0.020)$ \\
0.2 & $-0.052(-0.079)$ & $-0.023(-0.080)$ & $-0.018(-0.092)$ & $-0.005(-0.104)$ \\
0.3 & $-0.136(-0.172)$ & $-0.098(-0.164)$ & $-0.084(-0.176)$ & $-0.075(-0.187)$ \\
0.4 & $-0.225(-0.257)$ & $-0.177(-0.247)$ & $-0.150(-0.257)$ & $-0.127(-0.267)$ \\
0.5 & $-0.310(-0.342)$ & $-0.239(-0.329)$ & $-0.206(-0.336)$ & $-0.186(-0.349)$ \\
0.6 & $-0.390(-0.430)$ & $-0.313(-0.413)$ & $-0.270(-0.419)$ & $-0.240(-0.432)$ \\
0.7 & $-0.469(-0.514)$ & $-0.378(-0.492)$ & $-0.330(-0.502)$ & $-0.290(-0.513)$ \\
0.8 & $-0.539(-0.590)$ & $-0.440(-0.571)$ & $-0.375(-0.581)$ & $-0.331(-0.595)$ \\
0.9 & $-0.611(-0.672)$ & $-0.493(-0.645)$ & $-0.416(-0.662)$ & $-0.374(-0.680)$ \\
\hline
\end{tabular}

Table 6: MSE of $\hat{\rho}_{2 S R S}^{*}\left(\hat{\rho}_{2 M E R S S}^{*}\right)$

\begin{tabular}{ccccc}
\hline \multicolumn{5}{c}{$m$} \\
$\rho$ & 2 & 3 & 4 & 5 \\
\hline 0.1 & $0.044(0.029)$ & $0.060(0.024)$ & $0.058(0.018)$ & $0.056(0.014)$ \\
0.2 & $0.049(0.037)$ & $0.064(0.035)$ & $0.065(0.030)$ & $0.067(0.026)$ \\
0.3 & $0.071(0.062)$ & $0.081(0.060)$ & $0.081(0.055)$ & $0.080(0.053)$ \\
0.4 & $0.106(0.102)$ & $0.107(0.096)$ & $0.106(0.093)$ & $0.102(0.093)$ \\
0.5 & $0.155(0.157)$ & $0.143(0.148)$ & $0.132(0.143)$ & $0.127(0.144)$ \\
0.6 & $0.215(0.227)$ & $0.188(0.211)$ & $0.167(0.206)$ & $0.154(0.209)$ \\
0.7 & $0.289(0.308)$ & $0.235(0.285)$ & $0.205(0.284)$ & $0.180(0.287)$ \\
0.8 & $0.365(0.398)$ & $0.289(0.372)$ & $0.238(0.371)$ & $0.204(0.379)$ \\
0.9 & $0.454(0.504)$ & $0.341(0.464)$ & $0.269(0.472)$ & $0.230(0.485)$ \\
\hline
\end{tabular}

$\frac{\text { Table 7: Efficiency of } \hat{\rho}_{2 S R S}^{*} \text { W.r.t. } \hat{\rho}_{2 M E R S S}^{*}}{m}$

\begin{tabular}{ccccc}
$\rho$ & 2 & 3 & 4 & 5 \\
\hline 0.1 & 1.510 & 2.525 & 3.177 & 3.951 \\
0.2 & 1.323 & 1.830 & 2.159 & 2.438 \\
0.3 & 1.144 & 1.352 & 1.461 & 1.513 \\
0.4 & 1.031 & 1.110 & 1.135 & 1.105 \\
0.5 & 0.985 & 0.965 & 0.927 & 0.881 \\
0.6 & 0.945 & 0.892 & 0.814 & 0.737 \\
0.7 & 0.937 & 0.826 & 0.721 & 0.627 \\
0.8 & 0.918 & 0.778 & 0.642 & 0.539 \\
0.9 & 0.900 & 0.735 & 0.570 & 0.475 \\
\hline
\end{tabular}

\section{Acknowledgements}

The authors would like to thank the editor and the referee for their useful comments. Their comments were very helpful in revising the previous drafts of the paper. 
Table 8: Bias of $\hat{\lambda}_{2 S R S}\left(\hat{\lambda}_{2 M E R S S}^{*}\right)$

\begin{tabular}{ccccc}
\hline & \multicolumn{3}{c}{$m$} \\
$\rho$ & 2 & 3 & 4 & 5 \\
\hline 0.1 & $-0.003(-0.002)$ & $-0.002(-0.001)$ & $-0.005(0.008)$ & $-0.004(0.019)$ \\
0.2 & $0.000(0.016)$ & $-0.001(0.036)$ & $0.003(0.056)$ & $0.000(0.081)$ \\
0.3 & $0.000(0.037)$ & $-0.001(0.069)$ & $0.004(0.095)$ & $0.001(0.131)$ \\
0.4 & $0.005(0.060)$ & $-0.002(0.103)$ & $0.009(0.138)$ & $-0.004(0.193)$ \\
0.5 & $0.000(0.081)$ & $0.001(0.141)$ & $0.008(0.193)$ & $-0.004(0.239)$ \\
0.6 & $-0.002(0.103)$ & $0.001(0.170)$ & $0.006(0.228)$ & $0.004(0.289)$ \\
0.7 & $-0.004(0.120)$ & $-0.001(0.203)$ & $0.008(0.276)$ & $-0.007(0.341)$ \\
0.8 & $-0.001(0.142)$ & $-0.003(0.236)$ & $0.001(0.315)$ & $-0.003(0.396)$ \\
0.9 & $-0.006(0.170)$ & $-0.004(0.267)$ & $0.000(0.355)$ & $-0.002(0.453)$ \\
\hline
\end{tabular}

Table 9: MSE of $\hat{\lambda}_{2 S R S}\left(\hat{\lambda}_{2 M E R S S}^{*}\right)$

\begin{tabular}{ccccc}
\hline \multicolumn{5}{c}{$m$} \\
$\rho$ & 2 & 3 & 4 & 5 \\
\hline 0.1 & $0.500(0.491)$ & $0.333(0.332)$ & $0.250(0.249)$ & $0.199(0.210)$ \\
0.2 & $0.500(0.505)$ & $0.334(0.349)$ & $0.257(0.272)$ & $0.200(0.228)$ \\
0.3 & $0.501(0.515)$ & $0.330(0.363)$ & $0.254(0.285)$ & $0.200(0.245)$ \\
0.4 & $0.507(0.528)$ & $0.337(0.378)$ & $0.253(0.302)$ & $0.201(0.273)$ \\
0.5 & $0.500(0.540)$ & $0.333(0.387)$ & $0.258(0.319)$ & $0.196(0.301)$ \\
0.6 & $0.498(0.544)$ & $0.334(0.398)$ & $0.252(0.334)$ & $0.208(0.331)$ \\
0.7 & $0.501(0.545)$ & $0.332(0.403)$ & $0.259(0.351)$ & $0.200(0.351)$ \\
0.8 & $0.499(0.548)$ & $0.331(0.416)$ & $0.253(0.375)$ & $0.203(0.385)$ \\
0.9 & $0.496(0.548)$ & $0.329(0.424)$ & $0.242(0.385)$ & $0.197(0.427)$ \\
\hline
\end{tabular}

Table 10: Efficiency of $\hat{\lambda}_{2 M E R S S}^{*}$ w.r.t. $\hat{\lambda}_{2 S R S}^{*}$

\begin{tabular}{ccccc}
\hline \multicolumn{5}{c}{$m$} \\
$\rho$ & 2 & 3 & 4 & 5 \\
\hline 0.1 & 1.017 & 1.005 & 1.005 & 0.945 \\
0.2 & 0.990 & 0.956 & 0.946 & 0.876 \\
0.3 & 0.973 & 0.908 & 0.889 & 0.819 \\
0.4 & 0.960 & 0.892 & 0.838 & 0.739 \\
0.5 & 0.927 & 0.861 & 0.808 & 0.650 \\
0.6 & 0.917 & 0.839 & 0.754 & 0.628 \\
0.7 & 0.913 & 0.822 & 0.739 & 0.569 \\
0.8 & 0.911 & 0.797 & 0.675 & 0.526 \\
0.9 & 0.904 & 0.776 & 0.629 & 0.462 \\
\hline
\end{tabular}

\section{References}

Al-Odat, M. T., and Al-Saleh, M. F. (2001). A variation of ranked set sampling. Journal of Applied Statistical Science, 10, 137-146.

Al-Saadi, S. D., and Young, D. H. (1980). Estimation for the correlation coefficient in a 
bivariate exponential distribution. Journal of Statistical Computation and Simulation, 11, 13-20.

Al-Saleh, M. F., and Al-Hadrami, S. (2003a). Estimation of the mean of the exponential distribution using moving extremes ranked set sampling. Statistical Papers, 44, 367-382.

Al-Saleh, M. F., and Al-Hadrami, S. (2003b). Parametric estimation for the location parameter for symmetric distributions using moving extremes ranked set sampling with application to trees data. Environmetrics, 14, 651-664.

Al-Saleh, M. F., and Al-Kadri, M. (2000). Double ranked set sampling. Statistics and Probability Letters, 48, 205-212.

Al-Saleh, M. F., and Al-Omari, A. (2002). Multistage ranked set sampling. Journal of Statistical Planning and Inference, 102, 273-286.

Al-Saleh, M. F., and Diab, Y. A. (2009). Estimation of the parameters of downton's bivariate exponential distribution using ranked set sampling scheme. Journal of Statistical Planning and Inference, 139, 277-286.

Al-Saleh, M. F., and Samawi, H. M. (2010). On estimating the odds using moving extreme ranked set sampling. Statistical Methodology, 7, 133-140.

Al-Saleh, M. F., and Zheng, G. (2002). Estimation of bivariate characteristics using ranked set sampling. Australian and New Zealand Journal of Statistics, 44, 221232.

Bain, L. J. (1978). Statistical Analysis of Reliability and Life Testing Models: Theory and Methods. New York: Marcel Dekker.

Balakrishna, N., and Lai, C. D. (2009). Continuous Bivariate Distributions. New York: Springer.

Casella, G., and Berger, R. (2002). Statistical Inference (2nd ed.). California: Duxbury Press.

Chen, Z., Bai, Z., and Sinha, B. K. (2004). Ranked Set Sampling Theory and Applications. New York: Springer.

Diab, Y. A. (2006). Estimation of the parameters of Downton's bivariate exponential distribution using different ranked set sampling scheme. Unpublished master's thesis, Department of Statistics, Yarmouk University, Jordan.

Downton, F. (1970). Bivariate exponential distribution in reliability theory. Journal of the Royal Statistical Society, Series B, 32, 408-417.

He, Q., and Nagaraja, H. N. (2011). Correlation estimation in Downton's bivariate exponential distribution using incomplete samples. Journal of Statistical Computation and Simulation, 81, 531-546.

Iliopoulos, G. (2003). Estimation of parametric functions in Downton's bivariate exponential distribution. Journal of Statistical Planning and Inference, 117, 169-184.

McIntyre, G. A. (1952). A method for unbiased selective sampling using ranked sets. Australian Journal of Agricultural Research, 3, 385-390.

Moran, P. A. P. (1967). Testing for correlation between non-negative variates. Biometrika, 54, 385-394.

Samawi, H. M., Ahmed, M. S., and Abu-Dayyeh, W. (1996). Estimating the population mean using extreme ranked set sample. Biometrical Journal, 38, 577-586.

Stokes, S. L. (1977). Ranked set sampling with concomitant variables. Communications 
in Statistics-Theory and Methods, 6, 1207-1211.

Takahasi, K., and Wakimoto, K. (1968). On unbiased estimates of the population mean based on the sample stratified by means of ordering. Annals of the Institute of Statistical Mathematics, 20, 1-31.

Yang, S. S. (1977). General distribution theory of the concomitants of order statistics. The Annals of Statistics, 5, 996-1002.

Authors' addresses:

Ahmed Hanandeh

Department of Mathematical Sciences

University of Cincinnati

Cincinnati, OH 45221-0025

USA

E-mail: hanandaa@mail.uc.edu

Mohammad Fraiwan Al-Saleh

Department of Statistics

College of Sciences

Yarmouk University

Irbid- 21163

Jordan

E-mail: m-saleh@yu.edu.jo 